\title{
DENTSPLY und Sirona schließen definitiven Vertrag ab
}

\begin{abstract}
DENTSPLYInternational Inc.(„DENTSPLY“) und Sirona Dental Systems, Inc. („Sirona“) gaben bekannt, dass die Aufsichtsräte beider Unternehmen einstimmig dem definitiven Fusionsvertrag zugestimmt haben, der zum weltweit größten Hersteller für professionelle Dentalprodukte und -technologien führen wird. Dieser Zusammenschluss wird in einem fusionierten Unternehmen mit der größten Vertriebs- und Serviceinfrastruktur im Dentalbereich mit 15000 Mitarbeitern resultieren.
\end{abstract}

Total Solutions Provider with Leading Platforms

\begin{tabular}{|c|c|c|c|c|c|c|c|c|c|}
\hline \multicolumn{3}{|c|}{ Consumables } & \multicolumn{3}{|c|}{ Specialties } & \multicolumn{4}{|c|}{ Equipment } \\
\hline Preventive & Restorative & Prosthedes & Endodontics & Implants & Orthodontic & CADICAM & Imaging & $\begin{array}{l}\text { Treatment } \\
\text { Centers }\end{array}$ & instruments \\
\hline \multicolumn{10}{|c|}{ Strong Position } \\
\hline$\checkmark$ & $\checkmark$ & $\checkmark$ & $\checkmark$ & $\checkmark$ & $\checkmark$ & $\checkmark$ & $\checkmark$ & $\checkmark$ & $\checkmark$ \\
\hline \multicolumn{10}{|c|}{ Well-Established Brands } \\
\hline SIITUINE & Surefiys:R & CELTRA & PKOGHOER & XiVE' & 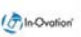 & CEREC & ОRTHOPHOS & TENEO & SIROLaser \\
\hline Cavitron' & Potodentphis & cercon' & Dovery & $\begin{array}{l}\text { ATLANTIS } \\
\text { ANKYLOS: }\end{array}$ & Senolloy & OMNICAM & GALILEOS & SINIUS & DAC \\
\hline NUPRO & MIDWEST & Portrair IPN & 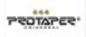 & ASTRATECH & $\mathrm{mtm}$ & CEREC MCX & schick & INTEGO & $\mathrm{r}_{4}$ \\
\hline
\end{tabular}

Broadened Range of Products and Solutions to Better Serve Customers

\section{DENSPLY SIITONa}

„Das ist ein aufregender Tag für beide Unternehmen, für Zahnärzte, Zahntechniker und für Patienten überall auf der Welt. Der Zusammenschluss der beiden weltweit führenden Unternehmen, die eine gemeinsame Kultur der Innovation teilen, fördert die Entwicklung von differenzierten, integrierten Lösungen für Zahnmediziner, Zahntechniker und Spezialisten, insbesondere in den stärksten Wachstumssegmenten der Dentalindustrie," sagt Jeffrey T. Slovin, President und Chief Executive Officer bei Sirona. „Durch das Zusammenführen von Sironas bewährten digitalen Lösungen und Produkten mit den führenden Verbrauchsgüterplattformen von DENTSPLY wird das umfangreichste Angebot an Dentallösungen geschaffen, das die Anforderungen der Kunden in allen Schlüsselsegmenten erfüllen wird. Ich freue mich darauf, den hoch erfahrenen Teams von Sirona und DENTSPLY auf unserem Weg zur globalen Digitalisierung der Zahnmedizin vorzustehen und Kunden sowie Patienten als ,THE Dental Solutions Company ' herausragende Lösungen anbieten zu können.“ „Wir freuen uns darauf, 2 Branchengrößen zusammenzubringen“, sagt Bret W. Wise, Chairman und Chief Executive Officer von DENTSPLY. „DENTSPLY SIRONA wird eine umfangreiche Produktpalette an Lösungen bereitstellen, die die Anforderungen der Zahnmedizin überall auf der Welt sowie die Ansprüche der Patienten besser erfüllen wird. Mit einem starken Finanzprofil, umfangreichen Produktangeboten und integrierten Lösungen, bringt sich DENTSPLY SIRONA in die einmalige Lage, die Zahnmedizin weltweit besser, schneller und sicherer zu gestalten. Ich freue mich darauf, mit Jeff und dem kombinierten Führungsteam an dieser Mission zu arbeiten.“

Das fusionierte Unternehmen wird DENTSPLY SIRONA heißen und an der NASDAQ unter dem Symbol XRAY gehandelt werden. Der globale Hauptsitz von DENTSPLY SIRONA wird in York, PA (USA) liegen, dem Standort des aktuellen Hauptsitz von DENTSPLY, während sich der internationale Hauptsitz in Salzburg, Österreich, befinden wird.

Nach einer Pressemitteilung von DENTSPLY International, USA - York, PA Sirona Dental GmbH, Bensheim 